\title{
19 Distinguishing Spinodal and Nucleation Phase Separation in Dewetting Polymer Films
}

\author{
O. K. C. Tsui*, B. Du, F. Xie, Y. J. Wang, H. Yan \\ and Z. Yang \\ Institute of Nano Science and Technology and Physics \\ Department, Hong Kong University of Science and \\ Technology, Clear Water Bay, Kowloon, Hong Kong
}

\section{INTRODUCTION}

It is a common experience that liquid films on non-wetting surfaces may dewet and break up into liquid droplets. Despite of its ordinariness, the physics of this phenomenon is not fully understood. Specifically, it cannot be resolved whether these thin films rupture by a spinodal mechanism or heterogeneous nucleation.

According to Cahn (1965), if the second derivative of a system's free energy as a function of the order parameter is less than zero (i.e. $G^{\prime \prime}(h)<0$ ), the system is unstable against spinodal decomposition. Under this circumstance, spontaneous fluctuations in the system order parameter may grow exponentially with time (Cahn, 1965). In particular, the fluctuation mode with wavevector, $q$, equals $q_{\mathrm{m}}=\sqrt{ }\left[G^{\prime \prime}(h) / \gamma\right]$ will grow the fastest, resulting in a characteristic wavevector $=q_{\mathrm{m}}$ in the morphology incurred in the initial stage of phase separation, though in the later stage coarsening of the morphology may occur (Chaikin and Lubensky, 1995) whereupon the characteristic wavevector will shift to smaller $q$. In apolar liquid films on a substrate, if the film thickness is less than $\sim 100 \mathrm{~nm}$, the free energy is mainly due to non-retarded van der Waals interactions (deGenees, 1985) so is of the form $-A / 12 \pi h^{2}$ per unit area, where $A$ is the Hamaker constant and $h$, i.e. the system order parameter, is the film thickness. It follows that those liquid films with $A<0$ are unstable against spinodal decomposition. From the foregoing, spinodal rupturing proceeds by exponential growth of the amplitude of the surface undulations in these films and the initial phase-separated state is a distribution of ridges and valleys before the dewetting film ripens into liquid beads. However, if $G^{\prime \prime}(h)$ is positive, the spinodal process will be suppressed. Nonetheless, so long as $G^{\prime}(h)$ is negative, the film will still undergo phase separation though by heterogeneous nucleation.

* Corresponding author. Email address: phtsui@ust.hk 
In distinguishing these two mechanisms in the rupturing of liquid (mostly polymer) films, the method often makes use of the known characteristics of the spinodal mechanism. Two characteristics are commonly used, including the occurrence of the bicontinuous structure in the phase separating morphology, which was predicted in Cahn's simulation, and the $q_{\mathrm{m}} \sim h^{-2}$ scaling, derivable from the above discussions. While the occurrence of a bicontinuous structure does provide unequivocal evidence for spinodal dewetting, cases in which the bicontinuous structure did not occur, but instead holes were formed are controversial. In these samples, the characteristic wavevector is determined from either the areal density of the holes, $N_{\mathrm{H}}$, appearing in the initial stage of the film rupturing or the areal density of droplets, $N_{\mathrm{d}}$, or polygons, $N_{\mathrm{p}}$, formed in the final stage. Compliance of $N_{\mathrm{H}}(h)$ or $N_{\mathrm{p}}(h)$ with the $h^{-4}$ scaling have been taken as an evidence of spinodal dewetting (Reiter, 1992), equivalent to the $q_{\mathrm{m}} \sim h^{-2}$ characteristic. On the other hand, Jacobs et al. (1998) pointed out that the holes had no spatial correlation and hence were unlikely due to spinodal growth of surface undulations. They further showed that the $N_{\mathrm{H}}(h) \sim h^{-4}$ scaling could be erroneously taken from an exponential dependence.

In this paper, we report a simple experiment by which we resolved this controversy unambiguously. In our approach, small height fluctuations were artificially introduced to the thin film samples by mechanically rubbing the film surface with a velvet cloth before dewetting takes place. Since the characteristic length of the dewetting pattern of polymer films that dewet by nucleation should depend on the density of surface defects introduced, but that of the films dewetting by a spinodal process should be relatively unaffected, one should be able to unambiguously identify which of the two dewetting mechanisms have in fact dominated in the rupturing of a given film simply by comparing its dewetting morphologies with and without the rubbing-induced defects.

\section{EXPERIMENT}

The system chosen for this study is polystyrene (PS) (molecular weight, $M_{\mathrm{w}}=13.7$ $\mathrm{K}$ Da and polydispersity index $=1.1$ ) spin-coated on silicon covered with a layer of $\sim 100 \mathrm{~nm}$ thick thermal oxide. The PS, purchased from Scientific Polymer Products (Ontario, NY), has a glass transition temperature, $T_{\mathrm{g}} \cong 99{ }^{\circ} \mathrm{C}$ from differential scanning calorimetry. To prepare the substrates, 4" diameter $\mathrm{Si}$ (100) wafers were subject to wet oxidation before cut into $1 \times 1 \mathrm{~cm}^{2}$ pieces, then cleaned as reported in an earlier publication (Wang et al., 2001). Upon spincoating with the PS, the samples were annealed at $100{ }^{\circ} \mathrm{C}$ under a $10^{-2}$ torr vacuum for $5 \mathrm{~h}$ to remove the residue solvent. No sign of dewetting could be found in the polymer films after annealing. To inscribe the topographical fluctuations, a piece of rayon cloth was rubbed against the film surface at a constant speed of $1 \mathrm{~cm} / \mathrm{s}$ under a normal pressure of $10 \mathrm{~g} / \mathrm{cm}^{2}$. The density of surface defects was controlled by changing the number of rubbings. The surface morphology of the films was characterized by a model SPA-300HV atomic force 
microscope (AFM) from Seiko Instruments (Chiba, Japan). Figure la shows the topographical image obtained from a PS film freshly rubbed by 15 times, and Figure $1 \mathrm{~b}$ shows its two-dimensional (2D) fast Fourier transformed (FFT) image, from which dominance of topographical features by rubbing is evident. The Fourier spectra of this sample and those rubbed by 3 and 10 times (obtained by radial averaging their 2D FFT images) were shown in Figure 1c. Evidently, the spectra due to different number of rubbings look the same. This strongly suggests

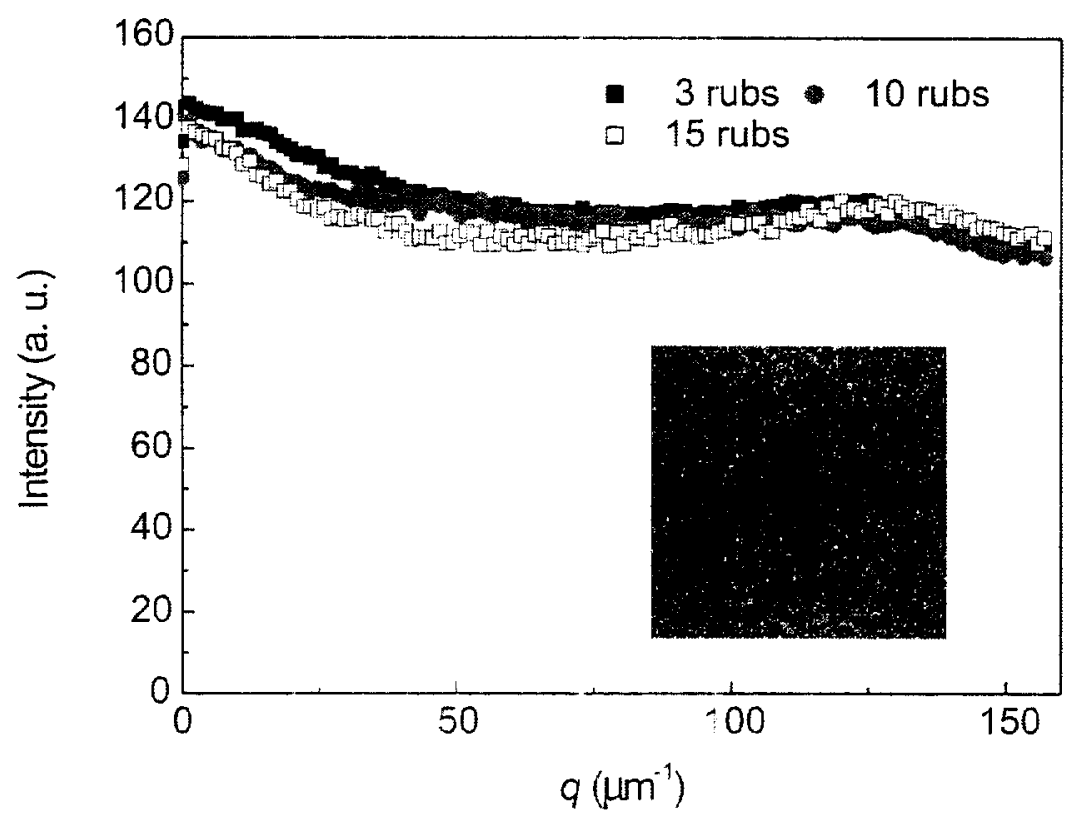

Figure 1 (a) AFM topographical image of a PS tilm freshly rubbed by 15 times. (b) The 2D FFT of the image shown in (a). (c) Fourier spectra of PS films rubbed 3,10 and 15 times, respectively.

that surface structures added to the films by individual rubbing are independent. It thus follows that the density of rubbing-induced surface defects should increase linearly with increasing $N$.

Dewetting experiments were carried out in air. The films were annealed at $180^{\circ} \mathrm{C}$ and $145^{\circ} \mathrm{C}$ from 10 to 60 mins. The different annealing temperatures were used to control the experimental times, and had been found to have no observable effect on the dewetting morphology. We deduce the characteristic wavevector, $q^{*}$, of the final dewetted patterns from the Fourier spectra. When the initial holes do not coalesce to form a network of polygons before breaking up into droplets, the dewetted patterns often display uniformly distributed liquid droplets (Figures 3 and 4 ). In that case, the square of the characteristic wavevector, $\left(q^{*}\right)^{2}$ is proportional to the areal density of the final liquid droplets, $N_{\mathrm{d}}$. On the other hand, if the holes do coalesce before they break up into liquid droplets, the dewetted patterns are composed of a network of polygons (Reiter, 1992). Then the square of the characteristic wavevector will instead be proportional to the areal density of the polygons, $N_{p}$. The change in physical meaning of $q^{*}$ simply 
reflects the different dewetted morphologies that were found in samples of different thickness. However, with either interpretations, $q^{*}$ serves to provide a measure of the characteristic length scale of the sample morphology for which we will compare between samples subject to different number of rubbings.

\section{RESULTS AND DISCUSSIONS}

Figure 2 shows $q^{*} v s . N$ for PS films of thicknesses $6.8 \mathrm{~nm}$ to $30 \mathrm{~nm}$. As seen, $q^{*}$ increases with decreasing $h$, consistent with previous results (Reiter, 1992; Jacobs, 1998). We further notice that $q^{*}$ of the samples with $h$ below $13.3 \mathrm{~nm}$ are independent of $N$ whereas $q^{*}$ of the thicker samples increases with increasing $N$. These data provide unequivocal evidence to the dominance of spinodal dewetting in samples with $h<13.3 \mathrm{~nm}$ but heterogeneous nucleation in samples with $h>13.3 \mathrm{~nm}$.

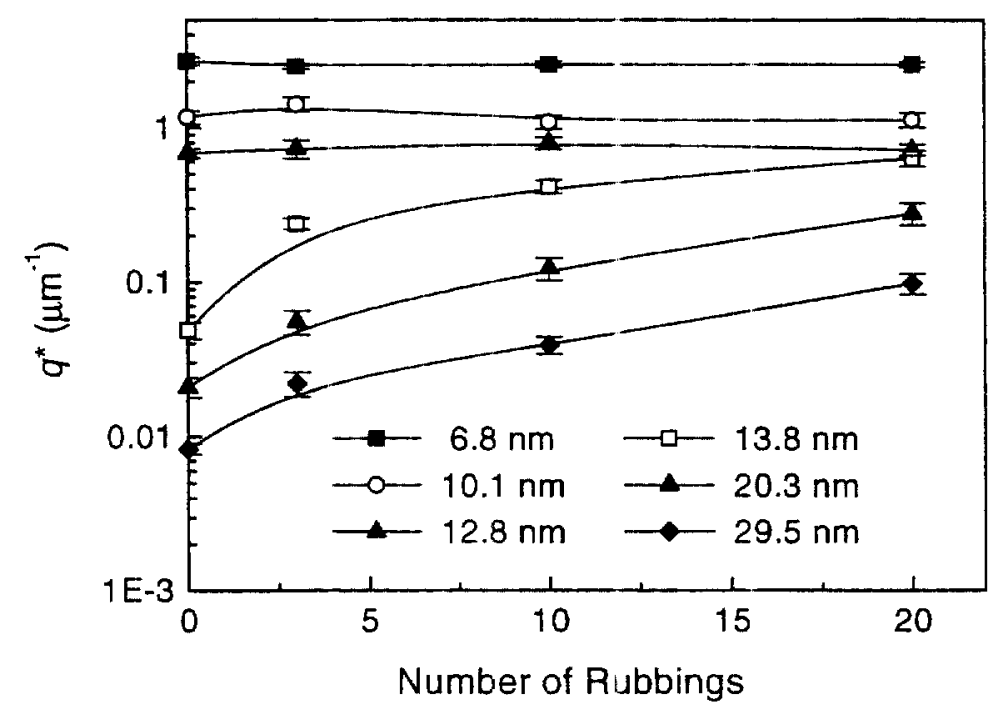

Figure 2 Characteristic wavevector, $q^{*}$ vs. $N$ for PS films with different thickness from $6.8 \mathrm{~nm}$ to $30 \mathrm{~nm}$. The solid lines are guides-to-the-eye.

To clarify the issue on the sample morphology obtainable from spinodal dewetting, we examine the evolution of dewetting in unrubbed films with thickness below $13.3 \mathrm{~nm}$. Figure 3 shows AFM topographical images obtained from a $6.8 \mathrm{~nm}$ thick PS film taken at different annealing times from 2 mins. to 33 mins. at $145^{\circ} \mathrm{C}$. As seen, regions of regular ridges and valleys first appeared after 2 mins. In 8 mins., the development into a bicontinuous structure becomes readily apparent. In Figure 4, we show the evolution of the rupturing morphology of a $8.9 \mathrm{~nm}$ thick film annealed at $145{ }^{\circ} \mathrm{C}$. As one can see, holes of uniform size first emerged and scattered randomly across the sample surface. They grew in 
size, and gradually more holes emerged with time. Until after $\sim 21$ mins., the holes filled up the whole area of the film whereupon the thin ribbons connecting the holes broke up into droplets.
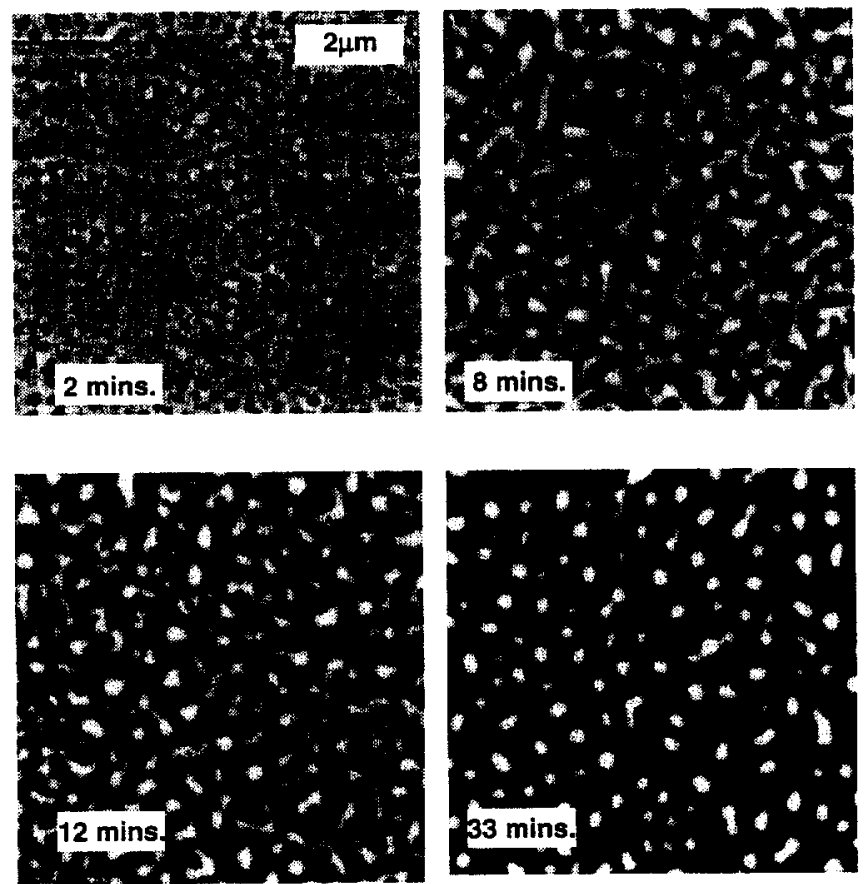

Figure 3 AFM topographical images of a $3.2 \mathrm{~nm}$ thick PS film taken after quenching at different annealing times from 2 mins. to 33 mins. at $145^{\circ} \mathrm{C}$.
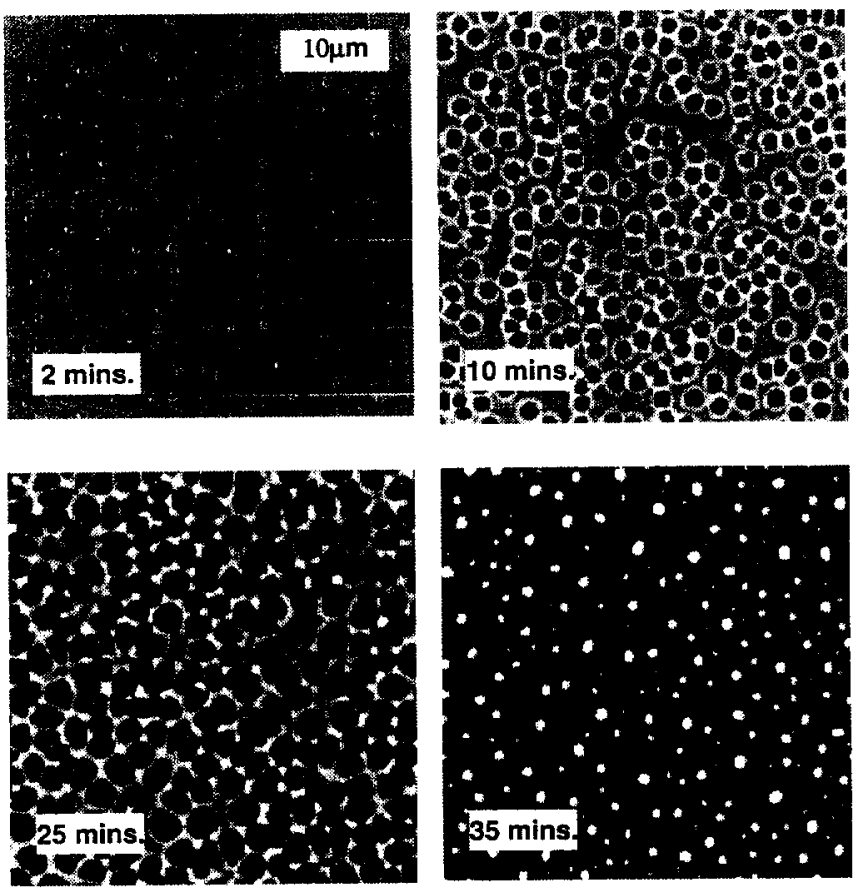

Figure 4 AFM topographical images of an $8.9 \mathrm{~nm}$ thick PS film dewetting at $145^{\circ} \mathrm{C}$. 
With results of Figures 3 and 4 , one may conclude about the dewetting morphologies formed in the early stage of spinodal dewetting. Basically, both the bicontinuous structure (Figure 3) and holes (Figure 4) may appear. The former forms in samples in the deep spinodal region whereas the latter appears in the thicker samples. This finding is reminiscent of the result from 3D nonlinear calculations by Sharma and Khanna (1998). These authors found that the morphology from spinodal dewetting can have either the bicontinuous structure or holes, depending on the form of $G(h)$. If $G(h)$ is such that $G^{\prime \prime}(h) \approx 0$, holes are formed, otherwise, the classic bicontinuous structure is preserved. While at this point there is not enough data to verify the origin of the transition at $h=13.3 \mathrm{~nm}$, a zero crossing of $G^{\prime \prime}(h)$ is certainly one possibility based on Sharma and Khanna's results. This is the question of a current study.

\section{ACKNOWLEDGEMENTS}

We acknowledge financial supports from the Institute of Nano Science and Technology and the Hong Kong University of Science and Technology through the Postdoctoral Matching Fund.

\section{REFERENCES}

Cahn, J. W., 1965, Phase separation by spinodal decomposition in isotropic systems. Journal of Chemical Physics, 42, pp. 93-99.

Chaikin, P. M. and Lubensky, T. C. 1995, Principles of Condensed Matter Physics, (Cambridge: Cambridge University Press).

deGenees, P. G., 1985, Dewetting: statics and dynamics. Review of Modern Physics, 57, 827-863.

Jacobs, K., Herminghaus, S. and Mecke, K. R., 1998, Thin liquid polymer films rupture via defects. Langmuir, 14, pp. 965-969.

Reiter, G., 1992, Dewetting of thin polymer films. Physical Review Letters, 68, pp. 75-78.

Sharma, A. and Khanna, R., 1998, Pattern formation in unstable thin liquid films. Physical Review Letters, 81, pp. 3463-3466.

Wang, X. P., Xiao, X. and Tsui, O. K. C., 2001, Surface viscoelasticity studies of ultrathin polymer films using atomic force microscopic adhesion measurements. Macromolecules, 34, pp. 4180-4185. 